\title{
Rehabilitation and COVID-19: the Cochrane Rehabilitation 2020 rapid living systematic review. Update as of July 31 $31^{\text {st }}, 2020$
}

Francesco Negrini ${ }^{1}$, Alessandro de Sire ${ }^{2,3}$, Elisa Andrenelli ${ }^{*}{ }^{*}$, Stefano Giuseppe Lazzarini ${ }^{5}$, Michele Patrini $^{5}$, Maria Gabriella Ceravolo4; The International Multiprofessional Steering Committee of Cochrane Rehabilitation REH-COVER action

1. IRCCS Istituto Ortopedico Galeazzi, Milan, Italy

2. Physical and Rehabilitative Medicine, Department of Health Sciences, University of Eastern Piedmont, Novara, Italy

3. Rehabilitation Unit, Mons. L. Novarese Hospital, Moncrivello, Vercelli, Italy

4. Department of Experimental and Clinical Medicine, "Politecnica delle Marche" University, Ancona, Italy

5. IRCCS Fondazione Don Gnocchi, Milan, Italy

The collective name International Multiprofessional Steering Committee of Cochrane Rehabilitation REH-COVER (REHabilitation for COVid-19: an Evidence-based Response) action includes the following contributors:

Carlotte Kiekens ${ }^{6}$, Chiara Arienti ${ }^{5}$, Maria Gabriella Ceravolo ${ }^{4}$, Pierre Côté ${ }^{7}$, Anne Cusick ${ }^{8}$, Francesca Gimigliano ${ }^{9}$, Allen Heinemann ${ }^{10}$, Jody-Anne Mills ${ }^{11,12}$, Farooq Rathore ${ }^{13}$, Marco Rizzi ${ }^{14}$, Geert Verheyden ${ }^{15}$, Margaret Walshe ${ }^{16}$, Stefano Negrini ${ }^{1,17}$

6. Spinal Unit, Montecatone Rehabilitation Institute, Imola (Bologna), Italy 
7. Faculty of Health Sciences, Ontario Tech University, Oshawa, Ontario, Canada

8. Discipline of Occupational Therapy, The University of Sydney, Sydney, Australia

9. Department of Mental and Physical Health and Preventive Medicine, University of Campania 'Luigi Vanvitelli', Napoli, Italy

10. Department of Physical Medicine and Rehabilitation, Northwestern University Feinberg School of Medicine, and Centre for Rehabilitation Outcomes Research, Shirley Ryan AbilityLab, Chicago, Illinois

11. Department of Noncommunicable Diseases, World Health Organization, Geneva, Switzerland

12. John Walsh Centre for Rehabilitation Research, Northern Clinical School, Faculty of Medicine and Health, University of Sydney, Sydney, Australia

13. Department of Rehabilitation Medicine, PNS Shifa Hospital, Karachi, Pakistan

14. Unit of Infectious Diseases, ASST Papa Giovanni XXIII Hospital, Bergamo, Italy

15. Department of Rehabilitation Sciences, KU Leuven - University of Leuven, Leuven, Belgium

16. Department of Clinical Speech and Language Studies, Trinity College Dublin, Dublin, Ireland

17. Department of Biomedical, Surgical and Dental Sciences, University "La Statale", Milan, Italy

Francesco Negrini and Alessandro de Sire equally contributed to this work. 
*Corresponding author: Elisa Andrenelli, Department of Experimental and Clinical Medicine, "Politecnica delle Marche" University, Ancona, Italy

\section{Abstract}

Background. This paper is the first update of the second edition of the rapid living systematic review on the latest scientific literature informing rehabilitation of patients with COVID-19 and/or describing consequences of the disease and its treatment, as they relate to limitations in functioning of rehabilitation interest.

Objectives. To report data of a systematic search performed on papers published in July 2020.

Methods. The methodology described in the second edition of the rapid living systematic review was applied to search eligible papers included in the databases between July $1^{\text {st }}, 2020$ and July $31^{\text {st }}, 2020$.

Results. Eight-hundred-ninety-two papers were identified through database searching (after removal of duplicates); of these, only 23 studies were included. According to OCEBM 2011 Levels of Evidence Table, they were Level 3 in $30.5 \%$ cases and Level 4 in $69.5 \%$. No RCT was found. Nineteen papers studied COVID-19 patients, assessed in the acute (10 studies), post-acute (8 studies) and chronic phase (one study). Four studies reported data on the impact of COVID-19 on subjects with pre-existing health conditions.

Conclusions. The current literature production still focuses more on describing all the possible aspects and complications of the pathology than on interventions or new organization models to 
deal with it. Albeit evidence on handling COVID-19 from a rehabilitative point of view is improving each month, further studies are still mandatory to report the role of rehabilitation in this scenario.

Keywords: COVID-19; Severe Acute Respiratory Syndrome Coronavirus 2; Coronavirus; Rehabilitation; Physical and Rehabilitation Medicine. 


\section{INTRODUCTION}

This work is the first update of the second edition of the rapid living systematic review ${ }^{1}$ aiming to present current evidence informing rehabilitation of patients with COVID-19 and/or describing consequences of the disease and its treatment, as they relate to limitations in functioning of rehabilitation interest (LFRI).

\section{METHODS}

This update follows the same methodology ${ }^{1}$ as the second edition of this rapid living systematic review. It adds eligible papers included in the databases between July $1^{\text {st }}, 2020$ and July $31^{\text {st }}$, 2020. The results report on the consolidated table of papers included in all editions of this living review is published in the Cochrane Rehabilitation REH-COVER website (https://tr.im/rr_dyn) ${ }^{2}$.

\section{RESULTS}

Out of 892 results identified through database searching (after removal of duplicates) for the period July $1^{\text {st }}, 2020$ to July $31^{\text {st }}, 2020,55$ full-text articles were assessed for eligibility; 32 papers were excluded because their content did not address any research question relevant to the scope of the present review ${ }^{1}$ (Figure 1). The remaining 23 included studies ${ }^{3-25}$ are presented in the Supplementary Table I (available on Cochrane Rehabilitation REH-COVER website: https://tr.im/rr07-20).

The majority of studies published in July 2020 were based, according to sampling site, in the following World Health Organization regions: Europe $(n=10)^{4,5,7,8,15,17,18,20,22,24}$, primarily in Italy $(n=6)^{4,5,7,15,18,20}$ the Americas (six in the United States of America $3,6,11,13,16,19$ and one in Canada ${ }^{12}$ ), 
Western Pacific (Singapore ${ }^{23}$, China $\left.^{9}, 14,25\right)$; and the Eastern Mediterranean region (Egypt ${ }^{21}$, Saudi Arabia $\left.^{10}\right)$.

\section{Evidence level of included studies}

Due to the heterogeneity of studies, a meta-analysis was not appropriate. According to the OCEBM 2011 Levels of Evidence Table ${ }^{26}$, the majority (69.5\%) were Level $4^{6,9-13,15-20,22-25}$, and the remainder $30.5 \%$ were Level $3^{3-5,7,8,14,21}$ (Table I). No RCT was found in this update

\section{Clinical characteristics of included studies}

Nineteen papers $(83 \%)^{4,6-12,14-20,22-25}$ studied COVID-19 patients, mostly assessed in the acute (10 studies) $)^{6,7,10,12,15-18,20,22}$ or post-acute phase (eight studies) $4,8,9,11,14,19,23,24$; only one study ${ }^{25}$ concerned patients in chronic phase. The remaining four studies ${ }^{3,5,13,21}$ reported data on the impact of COVID-19 on subjects with pre-existing health conditions (i.e., myasthenia gravis ${ }^{13}$, neuromuscular disease $(\mathrm{NMD})^{5}$, Parkinson disease $(\mathrm{PD})^{21}$, and spinal cord injuries and disorders $\left.(\mathrm{SCl} / \mathrm{D})^{3}\right)$.

Eight papers $(35 \%)^{3,4,6,8,11,16,19,23}$ provided data about a specific rehabilitation setting: three acute $^{6,16,19}$, one general post-acute ${ }^{11}$, three specialized post-acute ${ }^{3,4,23}$, and one home service ${ }^{8}$. Each included study presented sample and clinical outcome data that contributed evidence towards answering LFRI questions framing this review inquiry. Those relevant to this update are now presented. 


\section{Epidemiology - Impairment in respiratory structures and related functions}

Curci et al. ${ }^{4}$ investigated patients who were admitted to specialized post-acute rehabilitation settings after ICU care. This study reports that shortness of breath and dyspnoea are very common in these patients even during low-intensity activities. With regards to respiratory function (measured as fraction of inspired oxygen needed), the authors propose that personalized rehabilitative treatment is required for all post-acute COVID-19 patients using protocols aimed at reducing dyspnoea and improving muscle function.

Two papers studied lung function of COVID-19 patients after hospital discharge: one with a follow-up of 30 days $^{9}$ and one with a follow-up of three months ${ }^{25}$. Lung function abnormalities were reported in more than $50 \%$ of patients at 30 days, while $25 \%$ had abnormalities at 3 months.

\section{Epidemiology - Impairment in nervous system structures and related functions}

All together there were ten papers that presented evidence relating to nervous system impairment and related affected functions. Two case reports were not prima facie part of the COVID-19 presentation or complication. First, Piscitelli et al. described a young female developing lower limb tremor with variable frequency and amplitude during quarantine, probably of functional nature due to the trauma of diagnosis or to the isolation ${ }^{18}$. Second, a case report presented the complexities arising of COVID-19 in a patient with myasthenia gravis ${ }^{13}$.

The remaining eight case reports focused nervous system involvement in COVID-19 patients, either as the presenting clinical picture or as a complication occurring during the acute phase $^{6,10,12,15,17,20,22,24}$. Globally, the studies present: five patients with ischemic stroke $e^{6,20}$; one 
with hemorrhagic encephalopathy ${ }^{12}$; seven developing Guillain-Barré syndrome (GBS), or an acute polyradiculoneuritis suggestive of $\mathrm{GBS}^{10,15,17,24}$; and one developing a critical illness myopathy in the post-acute phase (65 days after hospital admission) ${ }^{22}$.

\section{Epidemiology - Any activity limitation and participation restriction}

Two cross-sectional studies used structured interviews (one by phone) to explore symptoms and rehabilitation needs of COVID-19 patients during hospital care ${ }^{14}(n=280)$ or after discharge ${ }^{8}$ ( $n=100)$. Hospitalized patients mainly complained of sleep disorders (63.6\%), decreased activity endurance (61.4\%), and respiratory dysfunction (57.9\%), while the main psychological dysfunctions included anxiety $(62.1 \%)$ and fear $(50.0 \%)^{14}$. In the study by Halpin et al ${ }^{8}$, one hundred patients were assessed 4-8 weeks after hospital discharge by rehabilitation professionals using a telephone screening tool also including a EQ-5D-5L telephone version. Fatigue was the most common reported symptom (72\% of people requiring ICU care in the acute phase, compared to $60.3 \%$ of those admitted to other wards), followed by breathlessness (65.6\% in ICU group; $42.6 \%$ in ward group) and psychological distress.

\section{Epidemiology - Impairment of any other body structure and function}

A cohort study showed that obese COVID-19 patients lose more lean mass and abdominal fat than non-obese patients ${ }^{7}$. The authors warn rehabilitators to be careful of sarcopenic obesity as corpulence could mask the loss of lean mass that can slow down the rehabilitation process. 


\section{Epidemiology - other papers}

A historical cohort study on 140 patients reported a 2.4 times higher COVID-19 related case fatality in veterans with SCI/D (19\%) than non SCI/D³.

Kirshblum et al. reported important data about the prevalence of COVID-19 in presumed COVID free patients at admission in rehabilitation in a high prevalence area ${ }^{11}: 6.8 \%$ of the patients were positive even without symptoms, and other $6.5 \%$ who tested negative developed symptomatology and became positive in the next 14 days.

Two studies ${ }^{5} 21$ investigated the effect of local public health preventative interventions of "lockdowns" on physical activity (PA) and quality of life (QoL) of patients with NMD and with PD, respectively, in comparison with healthy controls. Both studies interviewed subjects using the International Physical Activity Questionnaire finding out that, during the lockdown, people with neurological diseases experienced a greater PA reduction, than controls, with special regard to walking and moderate to vigorous exercise; besides, the results of SF-12 and PDQ39 questionnaires demonstrated an important QoL decrease, in NMD and PD subjects, respectively.

\section{Micro level - Interventions}

A single case report focused a rehabilitative intervention in a COVID-19 patient, who was mobilized using a robotic patient-guided suspension system ${ }^{23}$. The patient greatly improved, at discharge achieving independent ambulation and autonomy in most activities of daily living after 13 days. The authors propose that such systems, mainly implemented for neurologic 
rehabilitation, may be useful in respiratory rehabilitation of patients who face early desaturation and dyspnea due to severe COVID-19 infection.

\section{Meso level - Services}

Two historical cohort studies provided information on organizational aspects of rehabilitative services. The first reports the experience of an ICU for COVID-19 patients ${ }^{16}$, where a "Prone Team" was implemented, including rehabilitation therapists who already had expertise in mobilizing and positioning patients. The scope of this service was to provide proning to improve pulmonary function during mechanical ventilation, avoiding side effects due to incorrect mobilization or positioning of the patient such as nerve lesions or pressure sores. Criteria for Prone Therapy, including indications for starting and terminating prone ventilation, or returning to supine position were detailed. The prone team recorded preliminary information regarding process operations for scheduling and positioning purposes. The authors report that the team was involved in 934 position changes. These mostly involved three operators $(70 \%$ of the position changes) but in some cases, two (26\%), one (13\%), or four operators (11\%) were involved. The mean number of minutes for each position change was $20 \pm 9.15$ with a range of five to 80 minutes. Adverse events that may have occurred during mobility were not recorded.

Rosen et al. proposed an algorithm to identify patients with characteristics that could benefit from a physical therapy tele-rehabilitation service that was implemented in their hospital; this service was established to achieve the service goal of continuing patient treatment whilst maintaining staff safety through physical isolation from patients ${ }^{19}$. All the inpatients they treated either with tele-rehabilitation only (12) or with a combination of tele-rehabilitation and in-person 
physical therapy (21) met their physical therapy goals at the time of study conclusion and, where appropriate, were discharged.

\section{Discussion}

The paper included in this July update brings up some key points:

- The 23 included papers still show a wide heterogeneity in terms of methodological approaches: in particular, the outcome measures and assessment timing vary largely, limiting the possibility of secondary data analysis. Indeed, most papers presenting data on COVID-19 rehabilitation are case reports and case series describing the clinical features of COVID-19 patients $6,10,12,13,15,17,18,20,22,24$. However, there is an increase in the number and size of cohorts observed (either prospectively or retrospectively) to outline the natural history of the disease in the medium term $(11,16,19,25)$.

- Reports of acute neurologic complications in acute and post-acute phases of COVID-19 are multiplying and the rehabilitative community should be aware of the potential severe long-term disability due to those complications $s^{6,10,12,15,17,20,22,24}$.

- Two papers reported the clinical picture of post-acute COVID-19 patients finding that fatigue and breathlessness are very common and are recurrent symptoms ${ }^{8,14}$. This confirms speculation that post-COVID patients have ongoing physical and psychological symptoms that may benefit from rehabilitation interventions including exercise guidance, dietary instruction and, where available, traditional Chinese medicine therapy ${ }^{14}$.

- This review also contributes further evidence for clinical populations commonly engaged in rehabilitation who may be at heightened risk of COVID-19 complications given pre- 
existing conditions. For example, clinicians working with and advocacy groups engaged with SCI/D subjects should disseminate research information regarding the increased mortality risk in the case of COVID-19 infection in both outpatient and inpatient contexts. Strict observance of prevention measures and increased surveillance in inpatient and outpatient rehabilitation facilities may be warranted.

- During peak phases of the outbreak, given the high prevalence of positive asymptomatic patients ${ }^{11}$, any individual admitted to rehabilitation services should probably be considered and treated as potentially positive.

- The restriction of PA and walking activities together with the reduction in QoL, experienced during the lock-down, could theoretically speed up disease progression in a specific rehabilitation population. However, both studies investigating PA in $\mathrm{NMD}^{5}$ and PD patients ${ }^{21}$ did not use an objective measure of PA, such as the ones obtainable with an activity tracker, rather relying on self-reported information collected through the International Physical Activity Questionnaire. Given the risks outlined in the cited surveys, it could be worth developing tele-rehabilitation interventions that help frail people to maintain their fitness even when there are lockdown restrictions.

- The experience described by Rosen et al. ${ }^{19}$ could represent a useful starting point to implement tele-rehabilitation not only in the outpatient but also in the inpatient setting, to maximize safety for the operators while guaranteeing a functional service.

- Only one study described promising results obtained by a robot-assisted rehabilitation intervention at recovering gait autonomy in a post-acute COVID-19 patient ${ }^{23}$. The use of 
a robotic system could speed up recovery and reduce the length of stay of COVID-19 patients who lost their walking autonomy.

- The Prone Team proposed by Ng et al. ${ }^{16}$ was put under test during the peak phase of the COVID-19 pandemic. The authors described the experience in great detail so that it could be a model for other hospitals. However, the study lacks data about the side effects of proning, before and after the formation of the Prone Team, and further studies are needed to confirm their impact.

Taken all together, this month update confirms that current literature production still focus more on describing all the possible aspects and complications of the pathology than on interventions or new organization models to deal with it. However, more and more data are being collected, elaborated and peer-reviewed and knowledge on how to handle this new disease from a rehabilitative point of view is improving each month. 


\section{References}

1. Ceravolo MG, Arienti C, De Sire A, et al. Rehabilitation and Covid-19: the Cochrane Rehabilitation 2020 rapid living systematic review. Eur J Phys Rehabil Med. Published online July 24, 2020. doi:10.23736/S1973-9087.20.06501-6

2. Rehabilitation and COVID-19 - Evidence-based Response. Accessed August 5, 2020. /resources/cochrane-rehabilitation-versus-covid-19

3. Burns SP, Eberhart AC, Sippel JL, Wilson GM, Evans CT. Case-fatality with coronavirus disease 2019 (COVID-19) in United States Veterans with spinal cord injuries and disorders. Spinal Cord. Published online 2020. doi:10.1038/s41393-020-0529-0

4. Curci C, Pisano F, Bonacci E, et al. Early rehabilitation in post-acute COVID-19 patients: data from an Italian COVID-19 rehabilitation unit and proposal of a treatment protocol. A crosssectional study. Eur J Phys Rehabil Med. Published online July 15, 2020. doi:10.23736/S19739087.20.06339-X

5. Di Stefano V, Battaglia G, Giustino V, et al. Significant reduction of physical activity in patients with neuromuscular disease during COVID-19 pandemic: the long-term consequences of quarantine. J Neurol. Published online July 13, 2020. doi:10.1007/s00415-020-10064-6

6. Diaz-Segarra N, Edmond A, Kunac A, Yonclas P. COVID-19 Ischemic Strokes as an Emerging Rehabilitation Population: A Case Series. Am J Phys Med Rehabil. Published online July 15, 2020. doi:10.1097/PHM.0000000000001532

7. Gualtieri P, Falcone C, Romano L, et al. Body Composition Findings by Computed 
Tomography in SARS-CoV-2 Patients: Increased Risk of Muscle Wasting in Obesity. Int J Mol Sci. 2020;21(13). doi:10.3390/ijms21134670

8. Halpin SJ, Mclvor C, Whyatt G, et al. Post-discharge symptoms and rehabilitation needs in survivors of COVID-19 infection: a cross-sectional evaluation. J Med Virol. Published online 2020. doi:10.1002/jmv.26368

9. Huang Y, Tan C, Wu J, et al. Impact of coronavirus disease 2019 on pulmonary function in early convalescence phase. Respir Res. 2020;21(1):163. doi:10.1186/s12931-020-01429-6

10. Khalifa M, Zakaria F, Ragab Y, et al. Guillain-Barre Syndrome Associated with SARS-CoV-2 Detection and a COVID-19 Infection in a Child. J Pediatr Infect Dis Soc. Published online 2020. doi:10.1093/jpids/piaa086

11. Kirshblum SC, DeLauter G, Lopreiato MC, et al. Screening testing for SARS-CoV-2 upon admission to rehabilitation hospitals in a high COVID-19 prevalence community. PM R. Published online July 23, 2020. doi:10.1002/pmrj.12454

12. Krett JD, Jewett GAE, Elton-Lacasse C, et al. Hemorrhagic encephalopathy associated with COVID-19. J Neuroimmunol. 2020;346:577326. doi:10.1016/j.jneuroim.2020.577326

13. Kushlaf H. COVID-19 in muscle-specific kinase myasthenia gravis: A case report. Muscle Nerve. Published online July 13, 2020. doi:10.1002/mus.27020

14. Li Z, Zheng C, Duan C, et al. Rehabilitation needs of the first cohort of post-acute COVID19 patients in Hubei, China. Eur J Phys Rehabil Med. 2020;56(3):339-344. doi:10.23736/S19739087.20.06298-X 
15. Manganotti P, Bellavita G, D’Acunto L, et al. Clinical neurophysiology and cerebrospinal liquor analysis to detect Guillain-Barré syndrome and polyneuritis cranialis in COVID-19 patients: A case series. J Med Virol. Published online 2020. doi:10.1002/jmv.26289

16. Ng JA, Miccile LA, Iracheta C, et al. Prone Positioning of Patients With Acute Respiratory Distress Syndrome Related to COVID-19: A Rehabilitation-Based Prone Team. Phys Ther. Published online July 16, 2020. doi:10.1093/ptj/pzaa124

17. Pfefferkorn T, Dabitz R, von Wernitz-Keibel T, Aufenanger J, Nowak-Machen M, Janssen H. Acute polyradiculoneuritis with locked-in syndrome in a patient with Covid-19. J Neurol. 2020;267(7):1883-1884. doi:10.1007/s00415-020-09897-y

18. Piscitelli D, Perin C, Tremolizzo L, Peroni F, Cerri CG, Cornaggia CM. Functional movement disorders in a patient with COVID-19. Neurol Sci Off J Ital Neurol Soc Ital Soc Clin Neurophysiol. Published online 2020. doi:10.1007/s10072-020-04593-1

19. Rosen K, Patel M, Lawrence C, Mooney B. Delivering Telerehabilitation to COVID-19 Inpatients:A Retrospective Chart Review Suggests It Is a Viable Option. HSS ${ }^{\circledR}$. Published online July 16, 2020. doi:10.1007/s11420-020-09774-4

20. Saggese CE, Del Bianco C, Di Ruzza MR, Magarelli M, Gandini R, Plocco M. COVID-19 and Stroke: Casual or Causal Role? Cerebrovasc Dis Basel Switz. 2020;49(3):341-344. doi:10.1159/000509453

21. Shalash A, Roushdy T, Essam M, et al. Mental Health, Physical Activity, and Quality of Life in Parkinson's Disease During COVID-19 Pandemic. Mov Disord Off J Mov Disord Soc. 
2020;35(7):1097-1099. doi:10.1002/mds.28134

22. Tankisi H, Tankisi A, Harbo T, et al. Critical illness myopathy as a consequence of Covid-19 infection. Clin Neurophysiol. Published online 2020. doi:10.1016/j.clinph.2020.06.003

23. Tay SS, Neo EJ, Tan MM, Tan PL. Post-Critical Care COVID-19 Patient Benefits from a Robotic Patient-Guided Suspension System for Pulmonary Rehabilitation. Ann Acad Med Singapore. 2020;49(6):401-404.

24. Tiet MY, AlShaikh N. Guillain-Barré syndrome associated with COVID-19 infection: a case from the UK. BMJ Case Rep. 2020;13(7). doi:10.1136/bcr-2020-236536

25. Zhao $\mathrm{Y}$, Shang $\mathrm{Y}$, Song $\mathrm{W}$, et al. Follow-up study of the pulmonary function and related physiological characteristics of COVID-19 survivors three months after recovery. EClinicalMedicine. Published online July 15, 2020:100463. doi:10.1016/j.eclinm.2020.100463

26. OCEBM Levels of Evidence. CEBM. Published May 1, 2016. Accessed August 8, 2020. https://www.cebm.net/2016/05/ocebm-levels-of-evidence/ 
Table I. Level of evidence of the studies included in the present rapid living systematic review.

\begin{tabular}{|c|c|c|c|c|c|}
\hline & Level 1 & Level 2 & Level 3 & Level 4 & Total \\
\hline Epidemiology - Clinical presentation & 0 & 0 & $1(4.4 \%)$ & $5(21.7 \%)$ & $6(26.1 \%)$ \\
\hline Epidemiology - Prevalence & 0 & 0 & $5(21.7 \%)$ & 0 & $5(21.7 \%)$ \\
\hline $\begin{array}{l}\text { Epidemiology - Natural history / } \\
\text { Determining and modifying factors }\end{array}$ & 0 & 0 & $1(4.4 \%)$ & $8(34.7 \%)$ & $9(39.1 \%)$ \\
\hline Micro - Interventions (efficacy/harms) & 0 & 0 & 0 & $1(4.4 \%)$ & $1(4.4 \%)$ \\
\hline Meso Level & 0 & 0 & 0 & $2(8.7 \%)$ & $2(8.7 \%)$ \\
\hline Macro Level & 0 & 0 & 0 & 0 & 0 \\
\hline Total & 0 & 0 & 7 (30.5\%) & 16 (69.5\%) & $23(100 \%)$ \\
\hline
\end{tabular}

